\title{
Omega-6 and omega-3 polyunsaturated fatty acid levels are reduced in whole blood of Italian patients with a recent myocardial infarction: the AGE-IM study
}

\author{
Franca Marangoni ${ }^{\mathrm{a}, *}$, Giuseppina Novo ${ }^{\mathrm{b}}$, Giampiero Perna ${ }^{\mathrm{c}}$, Pasquale Perrone Filardi ${ }^{\mathrm{d}}$, \\ Salvatore Pirelli ${ }^{\mathrm{e}}$, Marco Ceroti ${ }^{\mathrm{f}}$, Andrea Querci ${ }^{\mathrm{f}}$, Andrea Poli ${ }^{\mathrm{a}}$ \\ ${ }^{a}$ NFI - Nutrition Foundation of Italy, Viale Tunisia 38, 20124 Milano, Italy \\ ${ }^{\mathrm{b}}$ Chair and Division of Cardiology, University of Palermo, Via del Vespro 129, 90127 Palermo, Italy \\ ${ }^{c}$ Cardiology and Coronary Care Unit - Ospedali Riuniti Ancona, Via Conca 71, Loc. Torrette, 60122 Ancona, Italy \\ ${ }^{\mathrm{d}}$ Department of Advanced Biomedical Sciences, Università Federico II, Napoli, Italy \\ e Cardiology and Coronary Care Unit, AO "Istituti Ospitalieri" di Cremona, Largo Priori 1, 26100 Cremona, Italy \\ ${ }^{\mathrm{f}}$ Molecular and Nutritional Epidemiology Unit, ISPO (Cancer Research and Prevention Institute) - Ponte Nuovo, Via delle Oblate 4, 50139 Firenze, Italy
}

\section{A R T I C L E I N F O}

\section{Article history:}

Received 31 July 2013

Received in revised form

30 October 2013

Accepted 18 November 2013

Available online 3 December 2013

\section{Keywords:}

Polyunsaturated fatty acids

Myocardial infarction

Fatty acid profile

Coronary heart disease risk

\begin{abstract}
A B S T R A C T
Objective: The relationship between whole blood fatty acids and myocardial infarction (MI) risk has not been analyzed in detail, especially in Mediterranean countries. The AGE-IM (Acidi Grassi Essenziali e Infarto Miocardico) study was planned to examine the relationships between MI, whole blood fatty acids and the diet in an Italian cohort.

Methods: 119 Patients with a recent MI and 103 control subjects were enrolled in the study. The whole blood fatty acid composition was determined; information on anthropometrics, biochemical parameters and blood pressure values were also obtained. Diet composition was assessed using a validated food frequency questionnaire from 86 cases and 72 controls.

Results: Total PUFA, omega-6 and omega-3 PUFA (as percentage of whole blood fatty acids) were significantly lower in MI patients than in matched controls, whereas saturated and monounsaturated fatty acids were higher in cases. MI infarction risk significantly and steadily decreased with increasing levels of total PUFA (OR: 0.14) and of total omega- 6 and omega-3 (OR: 0.15 and 0.37, respectively). No correlation was identified between dietary fats and MI risk or between whole blood fatty acid levels and dietary nutrients and fats.

Conclusion: Percentage levels of total PUFA, total omega-3 PUFA and total omega-6 PUFA are lower in MI patients than in matched control subjects in the AGE-IM cohort. These data support a favorable association not only of whole blood percentage levels of total omega-3, but also of total omega- 6 , with cardiovascular risk.
\end{abstract}

(c) 2013 Elsevier Ireland Ltd. All rights reserved.

\section{Introduction}

Dietary fats are known to affect coronary heart disease (CHD) risk [1]. Saturated and trans fatty acids (FA), notably, appear to increase such risk [2], while their replacement by monounsaturated (MUFA) and, especially, polyunsaturated (PUFA) fatty acids is considered to be protective $[3,4]$. A number of studies specifically demonstrate that high intakes of PUFA of both the omega- 3 and the omega- 6 series are protective against the probability to suffer from a CHD event $[5,6]$. The favorable effects of the two PUFA series are

\footnotetext{
* Corresponding author. Tel.: +392 76006271; fax: +392 76003514 .

E-mail address: marangoni@nutrition-foundation.it (F. Marangoni).
}

mediated by different mechanisms: for omega-3, a wide range of possible actions (anti-inflammatory, anti-aggregating and plaque stabilizing effects, improvement of endothelial function) has been described $[7,8]$ while the benefits of omega- 6 are largely attributed to their LDL lowering effect [9].

Some authors, on the other hand, hypothesize that the overall effect of omega- 6 consumption on CHD risk could be neutral or even negative, because of the higher susceptibility of these FA to oxidation, their potential pro-inflammatory effects, and their competition with omega-3 for the enzymatic pathway responsible for elongation and desaturation of the C-18 precursors of the two PUFA families, which might induce a reduction in the availability of long chain omega-3 PUFA and, in turn, of their 
cardioprotective activities [10]. Both omega-3 and omega-6 PUFA are actually essential to humans, who are unable to synthesize alpha-linolenic acid (ALA or 18:3 n-3 with 18 carbons and 3 double bonds) and can only partially synthesize the long chain omega-3 (EPA or eicosapentaenoic acid and DHA or docosahexaenoic acid) from ALA [11]. Linoleic acid (LA or 18:2 n-6, with 18 carbons and 2 double bonds), the precursor of the omega- 6 series, is also essential for mammalian cells which, unlike plant cells, lack the enzymes necessary for its de novo synthesis; LA and its longer chain metabolite, arachidonic acid, play relevant functional and structural roles in several specialized membranes in human cells [12]. Human need to ingest all these PUFA preformed with the diet.

The debate on the role of omega- 3 and omega- 6 PUFA in human diets and, specifically, on the relevance of their ratio in the prevention or promotion of degenerative diseases as CHD, has been ongoing for several years within the scientific community [13]. Recently, an International board of experts convened by FAO and WHO concluded that both (omega-6 and omega-3) PUFA families exert protective roles in the prevention of degenerative diseases and underscored the lack of substantial evidence on the role of the omega-3/omega-6 ratio within this context [14].

A precise assessment of the FA composition of the diet and, hence, of the actual dietary FA consumption, is unfortunately quite difficult. Usually, such data are collected using validated food frequency questionnaires (FFQ) and food diaries, giving information often imprecise and subjected to recollection bias [15]. Additionally, food composition databases are usually incomplete or only partially reliable as far as minor food components are concerned, as is the case of PUFA. This is due both to the limited availability of this information, but also to the highly variable content of these FA in similar foods from different origin or even in different samples of the same food [16].

The determination of the FA composition of circulating lipids (mainly in serum, plasma, red blood cells) has been proposed as an alternative approach able to provide information on the quality of dietary fats [14]. In fact, circulating FA levels reflect the dietary fat intake (especially of essential FA), even if they are also affected by specific features which are peculiar to each individual (age, gender, disease and consequently absorption efficiency, distribution, enzymatic metabolism, etc.) [17]. More recently, the direct analysis of whole blood FA, which is representative of both plasma and cell membrane lipids and provide an assessment of the FA status in the body, has been made possible by the availability of a simplified method for blood collection and adequate analytical procedures, providing a very reliable assessment of the FA status in the body $[18,19]$.

Harris and von Schacky found a negative association between the sum of EPA and DHA levels in red blood cell membranes (the socalled Omega-3 Index) and the risk for CHD death [20], while Imamura and coworkers observed higher circulating levels of long chain MUFA (22:1 and 24:1) in patients with incident chronic heart failure, as compared with control subjects [21].

In a case-control study carried out on more than 1500 U.S. subjects with a previous acute myocardial infarction or matched controls, significant differences were observed between the FA profiles of blood cell membranes in the two groups [22]. In particular, omega- 3 and omega-6 levels were higher in controls, whereas saturates, trans-unsaturates, and MUFA were higher in cases. However, the Authors of this study concluded that the prognostic and/or diagnostic value of FA markers with regard to CHD and the extent to which their modification by diet will alter such disease risk, warrants further study.

The AGE-IM study was designed to examine the relationships between myocardial infarction (MI), the FA composition of blood
Table 1

Description of MI patients and control subjects (mean, standard deviation).

\begin{tabular}{lll}
\hline & Cases & Controls \\
\hline N (Females) & $119(17)$ & $103(17)$ \\
Age $(\mathrm{y})$ & $55.9(8.1)$ & $55.1(8.6)$ \\
Currently smoking, $n(\%)^{*}$ & $73(61 \%)$ & $35(34 \%)$ \\
Weight $(\mathrm{kg})$ & $80.2(13.6)$ & $80.2(13.1)$ \\
BMI $\left(\mathrm{kg} / \mathrm{m}^{2}\right)$ & $27.7(4.5)$ & $26.6(3.9)$ \\
Waist $(\mathrm{cm})$ & $98.7(11.7)$ & $98.7(11.8)$ \\
Hip $(\mathrm{cm})$ & $101.8(9.9)$ & $102.9(10.7)$ \\
SBP $(\mathrm{mmHg})$ & $125(18)$ & $125(13)$ \\
DBP $(\mathrm{mmHg})$ & $76(14)$ & $78(9)$ \\
Glucose $(\mathrm{mg} / \mathrm{dL})^{* *}$ & $140(75)$ & $95(22)$ \\
Triglycerides $(\mathrm{mg} / \mathrm{dL})^{*}$ & $145(122)$ & $114(56)$ \\
Total cholesterol $(\mathrm{mg} / \mathrm{dL})$ & $202(41)$ & $196(37)$ \\
LDL cholesterol $(\mathrm{mg} / \mathrm{dL})$ & $129(33)$ & $47(14)$ \\
HDL cholesterol $(\mathrm{mg} / \mathrm{dL})^{*}$ & $43(12)$ & \\
\hline
\end{tabular}

${ }^{*} p \leq 0.05 ;{ }^{* *} p \leq 0.005$.

lipids, and the FA dietary intake in Italian MI patients and matched control subjects.

\section{Patients and methods}

AGE-IM (Acidi Grassi Ematici e Infarto Miocardico, or blood fatty acids and myocardial infarction) is a case-control study. The study protocol was approved by all the local Ethic Committees; informed consent to participate in the study was obtained from all participants in accordance with ethical standards. Cases were enrolled in five Italian Coronary Care Units (Ancona, Bologna, Cremona, Napoli, Palermo). Patients aged $18-70$ suffering a first acute MI in the preceding $24 \mathrm{~h}$ were considered for recruitment. MI diagnosis was made in accordance with International Classification of Diseases (ICD)-9 code 410 , and was based on typical history, electrocardiographic and enzyme changes and troponin plasma levels. Control subjects, matched by sex and age ( \pm 3 years), were recruited in the same hospitals, among patients admitted for general surgical procedures or dermatologic, orthopedic, ophthalmologic, ear nose and throat diseases other than cancers. The presence of hypercholesterolemia (total cholesterol $>300 \mathrm{mg} / \mathrm{dL}$ ) or previous statin use was considered as exclusion criterion for both cases and control subjects, together with the consumption of omega-3 based supplements or drugs, clinical history of stroke, peripheral artery disease or CHD. 119 MI patients and 103 control subjects were enrolled in the study between March 1, 2009 and March 30, 2011. 112 Matched pairs were obtained.

Anthropometric parameters (weight, height, waist circumference) were obtained from cases and control subjects, together with information about lifestyle (smoking habit, physical activity) and systolic and diastolic blood pressure values. Lipid profile and glycemia were determined with standard methods.

For FA determination, drops of whole blood were absorbed on a special strip of chromatography paper as already described $[18,19,23]$, immediately delivered to the laboratory and kept at $-20^{\circ} \mathrm{C}$ until analysis. FA methyl esters were prepared and analyzed by fast gas-chromatography as previously described [18,23]; results were expressed as percentage of total FA.

Information on average food intakes over the past year were obtained using a validated semiquantitative $\mathrm{FFQ}$ in the version currently used for the EPIC study in Italy [24], from 86 cases and 72 controls.

The Student $t$ test for paired differences was used to compare anthropometrics, biochemical parameters, whole blood FA levels and nutrient intakes between the two groups. Odds ratios (OR) for MI and the corresponding 95\% confidence intervals (CI) were computed using unconditional multiple logistic regression models 
comparing tertiles of each fatty acid classes/series. All regression equations included terms for age in quinquennia, sex, admission hospital, education, body mass index (BMI), smoking habit. Statistical analysis was performed using SAS 9.2 software; $p$ values $<0.05$ were considered statistically significant.

\section{Results}

The main characteristics of the study populations are summarized in Table 1. Current cigarette smoke was more frequent among cases than controls; moreover, plasma concentrations of glucose were higher in MI patients than in controls, also reflecting the prevalence of type 2 diabetes in the two groups (11\% and 6\% in cases and controls respectively). In MI patients triglycerides were higher too, while concentrations of HDL-cholesterol were lower. Triglyceride levels higher than $150 \mathrm{mg} / \mathrm{dL}$ were detected in $34 \%$ of cases and $22 \%$ of controls; HDL cholesterol concentrations lower than optimal ( $\leq 40 \mathrm{mg} / \mathrm{dL}$ for men and $\leq 50 \mathrm{mg} / \mathrm{dL}$ for women) were observed in $46 \%$ of cases and $31 \%$ of controls.

The FA percentage composition of whole blood from MI and control patients is shown in Table 2. Among saturated FA (SFA), palmitic acid was significantly higher in cases than in controls, while stearic acid levels were similar in the two groups. Oleic acid, which represents the large majority of MUFA, was more represented among cases than among controls. All the main PUFA, both of the omega- 3 and of the omega- 6 series, were found to be higher in blood samples obtained from controls than from cases. When the different FA classes were considered, total saturated FA (SFA) and MUFA were found to be higher and total PUFA lower among cases than among controls. The mean omega-6/omega-3 ratio was higher in cases. Exclusion of patients with elevated plasma TG levels, or with low HDL cholesterol levels, or with type 2 diabetes did not substantially affect the results; all the observed differences among cases and controls in whole blood FA were maintained, and maintained their statistical significance.

Table 3 shows the OR of MI according to subsequent tertiles of FA classes and series, together with results of tests for linear trend of tertiles. MI infarction risk significantly and steadily decreased with increasing levels of total PUFA (OR: $-73 \%$ and $-86 \%$ comparing the first to the second and to the third tertile, respectively). Similar trends were observed for the two PUFA series (omega-6 and omega-3) when separately analyzed. In particular, the OR associated to the highest tertile of blood levels of omega- 6

Table 2

Mean percentage levels of the main FA in whole blood from 112 matched pairs of MI patients and control subjects (mean, standard deviation).

\begin{tabular}{lrlrlc}
\hline & Cases & SD & Controls & SD & $p$ \\
\hline $16: 0$ & 28.48 & 2.47 & 26.97 & 2.64 & $<0.0001$ \\
$18: 0$ & 12.09 & 1.47 & 12.22 & 1.56 & 0.53 \\
$16: 1 \mathrm{n}-9$ & 1.36 & 0.59 & 1.33 & 0.48 & 0.68 \\
$18: 1 \mathrm{n}-9$ & 22.57 & 2.68 & 21.60 & 3.38 & 0.02 \\
$18: 2 \mathrm{n}-6$ & 15.51 & 2.14 & 16.18 & 2.57 & 0.03 \\
$18: 3 \mathrm{n}-6$ & 0.18 & 0.08 & 0.23 & 0.12 & 0.001 \\
$20: 3 \mathrm{n}-6$ & 1.19 & 0.30 & 1.26 & 0.29 & 0.09 \\
$20: 4 \mathrm{n}-6$ & 6.30 & 1.32 & 7.05 & 1.58 & 0.0001 \\
$22: 4 \mathrm{n}-6$ & 0.83 & 0.26 & 0.86 & 0.26 & 0.27 \\
$22: 5 \mathrm{n}-6$ & 0.17 & 0.08 & 0.20 & 0.08 & 0.04 \\
$18: 3 \mathrm{n}-3$ & 0.12 & 0.04 & 0.17 & 0.08 & $<0.0001$ \\
$20: 5 \mathrm{n}-3$ & 0.33 & 0.13 & 0.41 & 0.18 & 0.0003 \\
$22: 5 \mathrm{n}-3$ & 0.46 & 0.12 & 0.52 & 0.18 & 0.003 \\
$22: 6 \mathrm{n}-3$ & 1.35 & 0.52 & 1.56 & 0.55 & 0.004 \\
SFA & 44.58 & 2.82 & 43.40 & 3.30 & 0.005 \\
MUFA & 28.90 & 2.81 & 28.06 & 3.46 & 0.05 \\
PUFA & 26.39 & 3.27 & 28.43 & 4.19 & $<0.0001$ \\
Total n-6 & 24.17 & 2.92 & 25.78 & 3.71 & 0.0004 \\
Total n-3 & 2.26 & 0.68 & 2.66 & 0.85 & 0.0002 \\
n-6/n-3 & 11.38 & 2.71 & 10.46 & 2.82 & 0.01 \\
\hline
\end{tabular}

Table 3

Odds ratios of myocardial infarction and corresponding 95\% confidence intervals (CI) by tertiles of FA classes (percentage levels) in whole blood adjusted for education, body mass index (BMI), smoking habit and matching variables.

\begin{tabular}{|c|c|c|c|c|c|}
\hline & Tertiles & & OR & CI 95\% & Trend $p$ \\
\hline \multirow[t]{3}{*}{ SFA } & 1 & 40.81 & & & \\
\hline & 2 & 43.78 & 1.53 & $0.72-3.27$ & \\
\hline & 3 & 47.37 & 2.25 & $0.96-5.27$ & 0.064 \\
\hline \multirow{3}{*}{ MUFA } & 1 & 25.18 & & & \\
\hline & 2 & 28.36 & 1.15 & $0.50-2.64$ & \\
\hline & 3 & 31.90 & 1.95 & $0.77-4.90$ & 0.106 \\
\hline \multirow[t]{3}{*}{ PUFA } & 1 & 23.21 & & & \\
\hline & 2 & 27.45 & 0.27 & $0.10-0.79$ & \\
\hline & 3 & 31.58 & 0.14 & $0.05-0.40$ & 0.001 \\
\hline \multirow[t]{3}{*}{ Total n-6 } & 1 & 21.28 & & & \\
\hline & 2 & 25.03 & 0.2 & $0.06-0.60$ & \\
\hline & 3 & 28.62 & 0.15 & $0.05-0.44$ & 0.003 \\
\hline \multirow[t]{3}{*}{ Total n-3 } & 1 & 1.70 & & & \\
\hline & 2 & 2.35 & 0.52 & $0.21-1.28$ & \\
\hline & 3 & 3.33 & 0.37 & $0.15-0.90$ & 0.042 \\
\hline \multirow[t]{3}{*}{$n-6 / n-3$} & 1 & 7.99 & & & \\
\hline & 2 & 10.71 & 1.58 & $0.77-3.25$ & \\
\hline & 2 & 14.05 & 1.79 & $0.81-3.97$ & 0.092 \\
\hline
\end{tabular}

and omega-3, compared to the lowest tertile, were reduced by about 85\% (OR: 0.15; 95\%CI: $0.05-0.44$ ) and 63\% (OR: $0.37 ; 95 \% \mathrm{CI}$ : $0.15-0.90$ ) respectively. A significant $80 \%$ fall in MI risk (OR: 0.20 ; 95\% CI: 0.06-0.60) was found to be associated with omega- 6 levels in the second tertile, as compared to the first (lowest) while a nonsignificant OR reduction of about $48 \%$ (OR: 0.52 ; $95 \% \mathrm{CI}$ : $0.21-1.28$ ) was observed when the second and the first tertiles of omega- 3 distribution were compared.

No significant association was detected for the omega-6/omega3 ratio, or for SFA or MUFA levels, and MI risk in this model.

The nutrient composition of the diet, determined for $86 \mathrm{MI}$ patients and 72 control subjects (Table 4), resulted to be highly comparable in the two groups, except for starch intake, that was significantly higher among cases (173 g/day vs $148 \mathrm{~g} /$ day, respectively; $p=0.03$ ). No differences were observed, specifically, for total fat consumption or for the intake of animal or vegetable fats, or of SAT, MUFA or PUFA of the omega 6 or omega 3 series.

No significant relationship was identified between MI risk and the intake of different nutrients, or between the dietary intake of the 18-C FA (oleic, LA and ALA) and FA classes (expressed as percentage of total calories) and their levels in whole blood (data not shown).

\section{Discussion}

The main result of the AGE-IM study is represented by the different FA composition of whole blood in patients with a recent MI vs matched control subjects. In particular, PUFA of both the omega- 6 and the omega- 3 series are significantly less represented in whole blood from cases than from controls, whereas SFA and MUFA are lower in controls. This observation is substantially confirmed by the multivariate case/control analysis, showing a progressive and significant reduction of MI risk associated with increasing blood levels of total omega-6, and omega-3 PUFA. A statistically significant protective effect, specifically, was associated with both omega- 6 and omega- 3 percentage concentrations in the upper tertile and with omega- 6 levels in the intermediate tertile, as compared to the lowest tertiles of the same series. 
Table 4

Daily nutrient intake in the studied population. Data are expressed as $\mathrm{g} / \mathrm{day}$, if not otherwise indicated (mean, standard deviation).

\begin{tabular}{lcrrrr}
\hline & Cases & SD & Controls & SD & $p$ \\
\hline Subjects & \multicolumn{1}{c}{$(86)$} & & $(72)$ & & \\
& & & & & \\
Energy (kcal/day) & 2246 & 819 & 2101 & 851 & 0.28 \\
Water & 1180 & 597 & 1200 & 554 & 0.83 \\
Proteins (total) & 88.2 & 33.1 & 83.5 & 28.9 & 0.35 \\
Animal & 58.6 & 25.6 & 56.8 & 22.6 & 0.63 \\
Vegetable & 29.6 & 12.3 & 26.7 & 11.8 & 0.14 \\
Lipids (total) & 81.3 & 31.0 & 80.8 & 37.6 & 0.93 \\
Animal & 48.4 & 23.6 & 45.9 & 24.5 & 0.53 \\
Vegetable & 33.0 & 13.9 & 34.9 & 16.5 & 0.44 \\
Saturated & 28.6 & 12.6 & 28.1 & 14.6 & 0.79 \\
Monounsaturated & 38.7 & 14.5 & 38.9 & 17.4 & 0.92 \\
Oleic acid & 35.7 & 13.3 & 36.3 & 16.1 & 0.80 \\
Polyunsaturated & 9.3 & 3.9 & 9.2 & 4.4 & 0.83 \\
Linoleic acid & 7.1 & 3.0 & 7.0 & 3.6 & 0.88 \\
Alpha linolenic acid & 1.1 & 0.5 & 1.1 & 0.5 & 0.99 \\
Other PUFA & 1.1 & 0.5 & 1.0 & 0.7 & 0.56 \\
Cholesterol (mg/day) & 379.7 & 177.1 & 363.9 & 174.1 & 0.57 \\
Carbohydrates (total) & 284.5 & 124.0 & 257.3 & 112.9 & 0.15 \\
Starches & 175.6 & 84.7 & 148.3 & 73.5 & 0.03 \\
Soluble & 108.6 & 65.6 & 108.6 & 60.3 & 0.99 \\
Fiber & 18.0 & 7.2 & 18.2 & 7.2 & 0.89 \\
Alcohol & 13.4 & 19.8 & 10.5 & 13.8 & 0.29 \\
\hline & & & & &
\end{tabular}

Since omega-3 and omega-6 PUFA are essential FA, it is conceivable that these differences reflect - at least in part different dietary intakes. Indeed, such differences were not observed when data from validated FFQ were compared, suggesting that FFQ lack the sensitivity necessary to assess dietary components such as PUFA, present in small and variable amounts in foods; however, the impact of endogenous factors - different from dietary intake - possibly affecting PUFA metabolism and/or interesterification processes, and hence PUFA levels in whole blood, cannot be excluded.

As concerns MUFA, in agreement with Block et al., we observed higher oleic acid concentrations in MI cases as compared with controls [22], independently from the dietary intake of this FA, which is usually abundant in the Italian diet especially because of the large use of olive oil. When the OR associated to different oleic acid tertiles are compared, on the other hand, statistical significance is not reached.

The more elevated oleic acid levels in whole blood from MI patients may be associated, at least in part, to the higher mean triglycerides assessed in cases vs controls, due to the esterification of the same MUFA in this lipid class. However, as presented in the Results section, also after the exclusion of all subjects with more than $150 \mathrm{mg} / \mathrm{dL}$ triglycerides (37 cases and 17 controls), the difference between the two groups remain statistically significant (data not shown), suggesting the marginal role of triglyceridemia in the FA profile of the study population. Moreover, it should be emphasized that the specimen considered in this study, i.e. the whole blood, reflects the balanced proportion of FA pools in plasma and cells, and in particular of plasma phospholipids and red blood cell membranes, which are the main contributors to the FA amounts in blood [19]. The lack of correlation between MUFA (mainly oleic acid) percentage concentrations and the OR of MI is supported by data from case-control studies [22] and, when substituted for omega-6 PUFA, by a recent meta-analysis [25].

This study confirms, in whole blood and in an Italian population, the correlation between FA profile and CHD already reported in different specimens (erythrocyte membranes or plasma phospholipids) and in other population groups [22,26]. Taken together, these data suggest that the associations between different FA and cardiovascular risk are direct, rather than mediated by complex dietary patterns, which are likely to be significantly different among the various populations studied.

In addition to the protective effect of omega-3 PUFA, which is in accordance with several published data $[20,27]$, data from the AGEIM study support a favorable association between blood total omega-6 (mostly LA) percentage levels and cardiovascular risk. Although the LDL cholesterol-lowering effect of LA has been considered responsible for this protective association, together with the modulation of insulin sensitivity and systemic inflammation [28], no differences were observed in total and LDL cholesterol concentrations between MI patients and controls in association with differences in LA blood levels in this study. This might be due to the exclusion of patients with hypercholesterolemia during the recruitment; yet, it is in agreement with findings from other groups suggesting that lipid reduction alone is unlikely to fully explain the cardioprotective effect of omega-6 PUFA [29]. Interestingly, the dietary intake of omega-6 PUFA (9.3 g/day, corresponding to about $3.8 \%$ of total calories, very similar in the two study groups) was well below international recommendations (5$10 \%$ of total calories). This observation is consistent with recent Italian data showing that the total PUFA (mainly omega-6) intake is, on average, lower than $4 \%$ of total calories [30] and also lower than that assessed in other European Countries [31].

In the AGE-IM study, also the omega-6/omega-3 ratio was significantly different among cases and controls, in the absence, however, of significant association with the MI OR in multivariate analysis. The ratio between the two PUFA series may be strongly affected by their different variability (the range of percentage values for omega-3 is much larger than that of omega-6). Moreover, if both parameters are associated in a protective way with CHD risk, the use of ratios to compute their CHD protective roles is questionable, and may lead to incongruent or fully misleading results, as also underscored by the FAO/WHO document [14].

This study has some limitations. First of all, the case-control design does not allow establishing a clear cause-effect relationship between the observed FA differences and MI risk. Again, data collected via the use of FFQ can be of variable quality. Third, we cannot rule out an association between FA levels in cases and controls and parameters intrinsically associated with $\mathrm{MI}$ in our cohorts, such as cigarette smoke, glycemia, lipid alterations, etc.

Strong points mostly concern the concomitant collection of dietary records and blood fatty acid concentrations, which allowed comparing these parameters directly.

In conclusion, AGE-IM reports - in a population whose dietary fat of choice is olive oil - the association between high omega-3 and omega-6 PUFA percentage levels in whole blood and the reduction of cardiovascular risk and indicates that the intake of total PUFA is, on average, well below guidelines also in this Italian population [30-32]. If the observed correlations were indeed causal, the promotion of larger intakes of omega- 6 and omega- 3 PUFA would further contribute to coronary risk reduction in Italy.

\section{Acknowledgments}

The AGE-IM study was made possible by an unrestricted research grant from Bonomelli Montenegro. The funding source was not involved in study design, in the collection, analysis and interpretation of data, in the writing of the report and in the decision to submit the article for publication.

\section{References}

[1] Willett WC. Dietary fats and coronary heart disease. J Intern Med 2012;272: 13-24. http://dx.doi.org/10.1111/j.1365-2796.2012.02553.x. 
[2] Brouwer IA, Wanders AJ, Katan MB. Trans fatty acids and cardiovascular health: research completed? Eur J Clin Nutr 2013;67:541-7. http://dx.doi.org/ 10.1038/ejcn.2013.43.

[3] Mozaffarian D, Micha R, Wallace S. Effects on coronary heart disease of increasing polyunsaturated fat in place of saturated fat: a systematic review and meta-analysis of randomized controlled trials. PLoS Med 2010 Mar 23;7(3):e1000252. http://dx.doi.org/10.1371/journal.pmed.1000252.

[4] Mozaffarian D, Clarke R. Quantitative effects on cardiovascular risk factors and coronary heart disease risk of replacing partially hydrogenated vegetable oils with other fats and oils. Eur J Clin Nutr 2009;63:S22-33. http://dx.doi.org/ 10.1038/sj.ejcn.1602976.

[5] Willett WC. The role of dietary n-6 fatty acids in the prevention of cardiovascular disease. J Cardiovasc Med (Hagerstown) 2007;8:S42-5.

[6] De Caterina R. n-3 fatty acids in cardiovascular disease. N Engl J Med 2011;364:2439-50. http://dx.doi.org/10.1056/NEJMra1008153.

[7] Calder PC. n-3 Fatty acids and cardiovascular disease: evidence explained and mechanisms explored. Clin Sci (Lond) 2004;107:1-11.

[8] Kromhout D, Yasuda S, Geleijnse JM, Shimokawa H. Fish oil and omega-3 fatty acids in cardiovascular disease: do they really work? Eur Heart J 2012;33: 436-43. http://dx.doi.org/10.1093/eurheartj/ehr362.

[9] Mensink RP, Zock PL, Kester AD, Katan MB. Effects of dietary fatty acids and carbohydrates on the ratio of serum total to HDL cholesterol and on serum lipids and apolipoproteins: a meta-analysis of 60 controlled trials. Am J Clin Nutr 2003;77:1146-55.

[10] Ramsden CE, Hibbeln JR, Majchrzak SF, Davis JM. n-6 fatty acid-specific and mixed polyunsaturate dietary interventions have different effects on CHD risk: a meta-analysis of randomised controlled trials. Br J Nutr 2010;104: 1586-600. http://dx.doi.org/10.1017/S0007114510004010.

[11] Arterburn LM, Hall EB, Oken H. Distribution, interconversion, and dose response of n-3 fatty acids in humans. Am J Clin Nutr 2006;83:1467S-76S.

[12] Spector AA. Essentiality of fatty acids. Lipids 1999;34:S1-3.

[13] Harris WS. The omega-6/omega-3 ratio and cardiovascular disease risk: uses and abuses. Curr Atheroscler Rep 2006;8:453-9.

[14] Joint FAO/WHO Expert Consultation on Fats and Fatty Acids in Human Nutrition. Geneva: WHO; 10-14 November, 2008.

[15] Patel PS, Sharp SJ, Jansen E, et al. Fatty acids measured in plasma and erythrocyte-membrane phospholipids and derived by food-frequency questionnaire and the risk of new-onset type 2 diabetes: a pilot study in the European Prospective Investigation into Cancer and Nutrition (EPIC)-Norfolk cohort. Am J Clin Nutr 2010;92:1214-22. http://dx.doi.org/10.3945/ ajcn.2010.29182.

[16] Ariño A, Beltràn JA, Herrera A, Roncales P. Fish. In: Enc Human Nutr. pp: Elsevier Ltd.; 2005. pp. 247-56.

[17] Simopoulos AP. Genetic variants in the metabolism of omega- 6 and omega-3 fatty acids: their role in the determination of nutritional requirements and chronic disease risk. Exp Biol Med (Maywood) 2010 Jul;235(7):785-95. http://dx.doi.org/10.1258/ebm.2010.009298.

[18] Marangoni F, Colombo C, Galli C. A method for the direct evaluation of the fatty acid status in a drop of blood from a fingertip in humans: applicability to nutritional and epidemiological studies. Anal Biochem 2004;326:267-72.
[19] Risé P, Eligini S, Ghezzi S, et al. Fatty acid composition of plasma, blood cells and whole blood: relevance for the assessment of the fatty acid status in humans. Prostaglandins Leukot Essent Fatty Acids 2007;76:363-9.

[20] Harris WS, Von Schacky C. The omega-3 index: a new risk factor for death from coronary heart disease? Prev Med 2004;39:212-20.

[21] Imamura F, Lemaitre RN, King IB, et al. Long-chain monounsaturated Fatty acids and incidence of congestive heart failure in 2 prospective cohorts. Circulation 2013;127:1512-21. http://dx.doi.org/10.1161/CIRCULATIONAHA.112.001197. 1521e1-18.

[22] Block RC, Harris WS, Reid KJ, Spertus JA. Omega-6 and trans fatty acids in blood cell membranes: a risk factor for acute coronary syndromes? Am Heart ] 2008;156:1117-23. http://dx.doi.org/10.1016/j.ahj.2008.07.014.

[23] Marangoni F, Colombo C, Martiello A, et al. The fatty acid profiles in a drop of blood from a fingertip correlate with physiological, dietary and lifestyle parameters in volunteers. Prostaglandins Leukot Essent Fatty Acids 2007;76:8792.

[24] Pala V, Sieri S, Palli D, et al. Diet in the Italian EPIC cohorts: presentation of data and methodological issues. Tumori 2003;89:594-607.

[25] Jakobsen MU, O'Reilly EJ, Heitmann BL, et al. Major types of dietary fat and risk of coronary heart disease: a pooled analysis of 11 cohort studies. Am J Clin Nutr 2009;89:1425-32. http://dx.doi.org/10.3945/ajcn.2008.27124.

[26] Khaw KT, Friesen MD, Riboli E, et al. Plasma phospholipid fatty acid concentration and incident coronary heart disease in men and women: the EPIC Norfolk prospective study. PLoS Med 2012;9(7):e1001255. http://dx.doi.org/ 10.1371/journal.pmed.1001255.

[27] Mozaffarian D, Lemaitre RN, King IB, et al. Plasma phospholipid long-chain $\omega$ 3 fatty acids and total and cause-specific mortality in older adults: a cohort study. Ann Intern Med 2013;158:515-25. http://dx.doi.org/10.7326/00034819-158-7-201304.

[28] Czernichow S, Thomas D, Bruckert E. n-6 Fatty acids and cardiovascular health: a review of the evidence for dietary intake recommendations. Br J Nutr 2010;104:788-96. http://dx.doi.org/10.1017/S0007114510002096.

[29] Harris WS, Mozaffarian D, Rimm E, et al. Omega-6 fatty acids and risk for cardiovascular disease: a science advisory from the American Heart Association Nutrition Subcommittee of the Council on Nutrition, Physical Activity, and Metabolism; Council on Cardiovascular Nursing; and Council on Epidemiology and Prevention. Circulation 2009;119:902-7. http://dx.doi.org/ 10.1161/CIRCULATIONAHA.108.191627.

[30] Sette S, Le Donne C, Piccinelli R, et al. The third Italian National Food Consumption Survey, INRAN-SCAI 2005-06 - part 1: nutrient intakes in Italy. Nutr Metab Cardiovasc Dis 2011;21:922-32. http://dx.doi.org/10.1016/ j.numecd.2010.03.001.

[31] Freisling H, Fahey MT, Moskal A, et al. Region-specific nutrient intake patterns exhibit a geographical gradient within and between European countries. J Nutr 2010;140:1280-6. http://dx.doi.org/10.3945/jn.110.121152.

[32] Sofi F, Innocenti G, Dini C, et al. Low adherence of a clinically healthy Italian population to nutritional recommendations for primary prevention of chronic diseases. Nutr Metab Cardiovasc Dis 2006;16:436-44. 